\title{
Vimentin expression and the influence of Matrigel in cell lines of head and neck squamous cell carcinoma
}

\section{Aluana Maria da Costa Dal Vechio(a) \\ Fernanda Salgueiredo Giudice ${ }^{(a)}$ \\ Felipe Fornias Sperandio(a) \\ Andrea Mantesso(a) \\ Décio dos Santos Pinto Junior ${ }^{(a)}$}

(a) Department of Oral Diagnosis and Oral Pathology, School of Dentistry, University of São Paulo, São Paulo - SP - Brazil.
Conflict of interest statement:

The authors declare that they have no conflict of interest.

\section{Corresponding author:}

Aluana Maria da Costa Dal Vechio

University of São Paulo

Department of Oral Pathology

Av. Prof. Lineu Prestes, 2227

CEP: 05508-900

São Paulo - SP - Brazil

E-mail: lumadal@usp.br

Received for publication on Nov 17, 2010 Accepted for publication on May 03, 2011

\begin{abstract}
Vimentin is a cytoeskeletal intermediate filament protein commonly observed in mesenchymal cells; however, it can also be found in malignant epithelial cells. It is demonstrated in several carcinomas, such as those of the cervix, breast and bladder, in which it is widely used as a marker of the epithelial to mesenchymal transition that takes place during embryogenesis and metastasis. Vimentin is associated with tumors that show a high degree of invasiveness, being detected in invasion front cells. Its expression seems to be influenced by the tumor microenvironment. The aim of this study was to evaluate vimentin expression in head and neck squamous cell carcinoma (HNSCC) cell lines, and to investigate the contribution of the microenvironment to its expression. HNSCC cell lines (HN6, HN30 and HN31) and an immortalized nontumorigenic cell line $(\mathrm{HaCaT})$ were submitted to a three-dimensional assay with Matrigel. Cytoplasmatic staining of the HN6 cell line cultured without Matrigel and of the HN30 and HN31 cell lines cultured with Matrigel was demonstrated through immunohistochemistry. Western Blotting revealed a significant decrease in vimentin expression for the HN6 cell line and a significant increase for the HN30 and HN31 cell lines cultured with Matrigel. The results suggest that vimentin can be expressed in HNSCC cells and its presence is influenced by the microenvironment of a tumor.
\end{abstract}

Descriptors: Carcinoma, Squamous Cell; Vimentin; Extracellular Matrix.

\section{Introduction}

Head and neck squamous cell carcinoma (HNSCC) is the sixth most common cancer worldwide. It represents $90 \%$ of all head and neck malignancies and causes more deaths than any other oral disease. ${ }^{1,2}$

It is known that cancer progression is marked by genetic changes that control the interaction between the cells and the extracellular matrix. Thus, some signaling pathways can be stimulated, or even suppressed, thereby inducing cell proliferation ${ }^{3,4}$ and replacing the epithelial phenotype of intercellular tight junctions and polarity by a more mesenchymal phenotype with reduced cell-cell adhesions, altered shape, expression of mesenchymal cellular markers and enhanced cell motility. ${ }^{5-7}$

These morphological changes define the epithelial-mesenchymal transition (EMT), which is recognized as a hallmark of tumor progression, that characterizes highly invasive and metastatic carcinomas. ${ }^{7,8}$ 
Proteins related to invasion, cell proliferation and metastasis are being evaluated exhaustively. Vimentin is a type III intermediate filament protein, present in most mesenchymal cells, that is expressed when tumor cells undergo EMT.9-11 This protein contributes to the aggressive pattern of cancer cells. It is widely studied in carcinomas of the breast, cervix and bladder, ${ }^{12,13}$ and has been suggested to be a predictor of the recurrence and invasive potential of prostate cancer cells. ${ }^{14}$

The extracellular matrix controls cell behavior and influences cell development, migration, proliferation, morphology and function, thereby creating an anchor for several other structures..$^{15}$ It is known that neoplastic cells have abnormal interactions with their environments. ${ }^{16}$

Matrigel is a reconstituted basement membrane that biologically mimics this environment and stimulates cell differentiation. ${ }^{17}$ Although the twodimensional model is the usual method used for in vitro cell culturing, and provides a convenient and fast means for research, it represents a limited tool for analyzing specific tissue functions and signaling pathways. ${ }^{18}$

Therefore, the purpose of the present investigation was to evaluate vimentin expression in HNSCC cell lines and to investigate the influence of Matrigel upon its expression.

\section{Methodology}

This study was approved by the Ethics Committee of the School of Dentistry of the University of São Paulo (protocol 134/05). All experiments were performed in triplicate.

\section{Cell lines and culture conditions}

The HNSCC cell lines [HN6 (base of tongue), HN30 (pharynx), HN31 (lymph node metastatic cells) $]^{19}$ and $\mathrm{HaCaT}$, an immortalized nontumorigenic human skin keratinocyte cell line, were cultured in DMEM (Sigma-Aldrich, St. Louis, USA) supplemented with $10 \%$ fetal bovine serum (Gibco, Grand Island, USA), and 1\% antibiotic-antimycotic solution (Sigma-Aldrich, St. Louis, USA). The cells were maintained in a $5 \% \mathrm{CO}_{2}$-humidified incubator.

\section{Immunohistochemistry and hematoxylin- eosin staining}

For two-dimensional assay, the cell lines were cultured in $75 \mathrm{~cm}^{2}$ culture flasks until reaching $80 \%$ confluence. Then, they were removed (trypsinization - Trypsin Tryple Express - Gibco, Grand Island, USA), fixed in $4 \%$ paraformaldehyde (Synth, Diadema, Brazil) and dehydrated in a graded ethanol series (Synth, Diadema, Brazil). HistoGel ${ }^{\mathrm{TM}}$ (Richard-Allan Scientific, Kalamazoo, USA) was added and the samples were incubated in a graded ethanol series, ethanol/xylene (1:1), xylene (Synth, Diadema, Brazil) and paraffin, to obtain cell blocks.

In terms of a three-dimensional cell culture model, the cell lines were seeded in $75 \mathrm{~cm}^{2}$ culture flasks, cultivated until reaching $80 \%$ confluence, removed (trypsinization - Trypsin Tryple Express - Gibco, Grand Island, USA) and resuspended in 1:3 Matrigel (BD Matrigel ${ }^{\mathrm{TM}}$ Matrix - cat. Number 356231 Two Oak Park, Bedford, USA) for 72 hours. Then, the samples were fixed following the same protocol as in the two-dimensional assay.

Three- $\mu \mathrm{m}$ thick tissue sections were deparaffinized and re-hydrated in a graded ethanol series (Synth, Diadema, Brazil). One slide was stained with Hematoxylin and Eosin (Synth, Diadema, Brazil). Vimentin expression was detected by the avidin-biotin peroxidase method. Sections were deparaffinized and, for antigen retrieval, were treated with citric acid $(10$ mM, pH 6.0 - Sigma-Aldrich, St. Louis, USA) by heating at $95{ }^{\circ} \mathrm{C}$ for $30 \mathrm{~min}$. Endogenous peroxidase was then blocked with hydrogen peroxide and sections were incubated with anti-vimentin (cloneV9, concentration of 1:200, Dako, Carpinteria, USA). Diaminobenzidine (Dako, Carpinteria, USA) was used as the chromogen, followed by counterstaining with Mayer's hematoxylin (Synth, Diadema, Brazil). Negative controls were obtained by omitting primary antibodies.

\section{Western blotting}

The cells cultured without Matrigel were harvested in $58 \mathrm{~cm}^{2}$ culture dishes, cultivated until reaching $80 \%$ confluence and washed three times with cold 1X PBS (Sigma-Aldrich, St. Louis, USA). They were lysed with lysis buffer $[50 \mathrm{mmol} / \mathrm{L}$ Tris- $\mathrm{HCl}$ 
(pH 7.4), $1 \mathrm{mmol} / \mathrm{L}$ EDTA, $150 \mathrm{mmol} / \mathrm{L} \mathrm{NaCl}, 1 \%$ Triton X-100, 1\% DOC, $0.1 \%$ SDS (Sigma-Aldrich, St. Louis, USA) with freshly added protease inhibitor cocktail (Sigma-Aldrich, St. Louis, USA)] at $4{ }^{\circ} \mathrm{C}$ for $20 \mathrm{~min}$ and scraped so the lysate could be collected in a microfuge tube. The sample was cleared by centrifugation at $13,000 \mathrm{rpm}$ for $25 \mathrm{~min}$ at $4{ }^{\circ} \mathrm{C}$, and the supernatant was collected and stored at $-80{ }^{\circ} \mathrm{C}$ for posterior quantification. Protein concentrations of all samples were determined using the Pierce BCA method (Pierce Biotechnology, Rockford, USA), following the manufacturer's protocol.

In the case of the three-dimensional cell culture model, the cell lines were cultivated in $75 \mathrm{~cm}^{2}$ culture flasks until reaching $80 \%$ confluence, removed (trypsinization - Trypsin Tryple Express - Gibco, Grand Island, USA) and resuspended in 1:3 Matrigel for 72 hours. Cold 1X PBS (Sigma-Aldrich, St. Louis, USA) was added, the sample was cleared by centrifugation at $13,000 \mathrm{rpm}$ for $25 \mathrm{~min}$ at $4{ }^{\circ} \mathrm{C}$ and the supernatant was discarded. This step was repeated thrice. The cell pellet was incubated in an ice-cold lysis buffer for $20 \mathrm{~min}$ at $4{ }^{\circ} \mathrm{C}$ and clarified by centrifugation at $13,000 \mathrm{rpm}$ for $25 \mathrm{~min}$ at $4{ }^{\circ} \mathrm{C}$. Supernatants (total cell lysate) were collected and stored at $-80{ }^{\circ} \mathrm{C}$ for posterior quantification, as previously stated.

For Western blotting analysis, $20 \mu \mathrm{g}$ of protein were loaded onto $10 \%$ polyacrylamide gels and transferred to a polyvinylidene fluoride membrane (PVDF membrane) (Biorad Laboratories, Hercules, USA). Non-specific binding sites on the membrane were blocked by incubation in a blocking buffer $(5 \%$ non-fat dry milk, $1 \%$ Tween-20 in $20 \mathrm{mmol} / \mathrm{L}$ Tris buffered saline ( $\mathrm{pH} 7.6$ - Sigma-Aldrich, St. Louis, USA) for 2 hours. Blots were probed overnight with primary antibody vimentin (clone V9, concentration of 1:200, Sigma-Aldrich, St. Louis, USA), or for 2 hours with primary antibody beta-actin (the ACTB gene is one of six different actin isoforms which have been identified in humans) (Sigma-Aldrich, St. Louis, USA), and for 90 minutes with peroxidase-conjugated secondary antibody (goat anti-mouse IgGHRP, Santa Cruz Biotechnology, Santa Cruz, USA). A bound antibody was detected by a colorimetric method using an Opti 4CN kit (Biorad Laborato- ries, Hercules, USA). Relative band intensities were determined using densitometry software (NIH, Image J 1.42, Bethesda, USA). Statistical analyses, to compare each group, were estimated using Student's $t$-test. Data were considered significant if the $P$ value was $<0.05$.

\section{Results Histological findings}

The HaCaT cell line, cultured either with or without Matrigel, demonstrated a non-pleomorphic, monotonous appearance (Figures $1 \mathrm{~A}$ and $1 \mathrm{~B}$ ). On the other hand, the HNSCC cell lines cultured without Matrigel exhibited nuclear pleomorphism, discrete cell size variation and absence of mitosis (Figure 1C). The same morphological characteristics were found when these cell lines were cultivated with Matrigel, although they were more evident and some mitotic cells could be identified (Figure 1D).

\section{Vimentin immunohistochemical expression}

Vimentin expression was not observed in the HaCaT, HN30 and HN31 cell lines cultured without Matrigel. In contrast, scattered cells from the HN6 cell line showed cytoplasmic positivity (Figure 1E). When cultured with Matrigel, the HaCaT and HN6 cell lines demonstrated no vimentin expression while the HN30 and HN31 cell lines showed immunopositivity (Figures 1F and 1G).

\section{Vimentin intracellular levels}

Western blotting confirmed the results shown by immunohistochemistry. A significant decrease in vimentin expression levels for the HN6 cell line $(\mathrm{p}=0.022)$, and a significant increase for the HN30 $(\mathrm{p}=0.027)$ and HN31 $(\mathrm{p}=0.030)$ cell lines cultured with Matrigel, were noted. HaCaT showed no expression when cultured with Matrigel. Additionally, only the HN6 cell line showed evident vimentin expression levels when cultured without Matrigel (Figure 2).

\section{Discussion}

Many biological processes such as motility, invasion, cancer progression and epithelial cell differentiation may be better mimicked in three-dimensional 

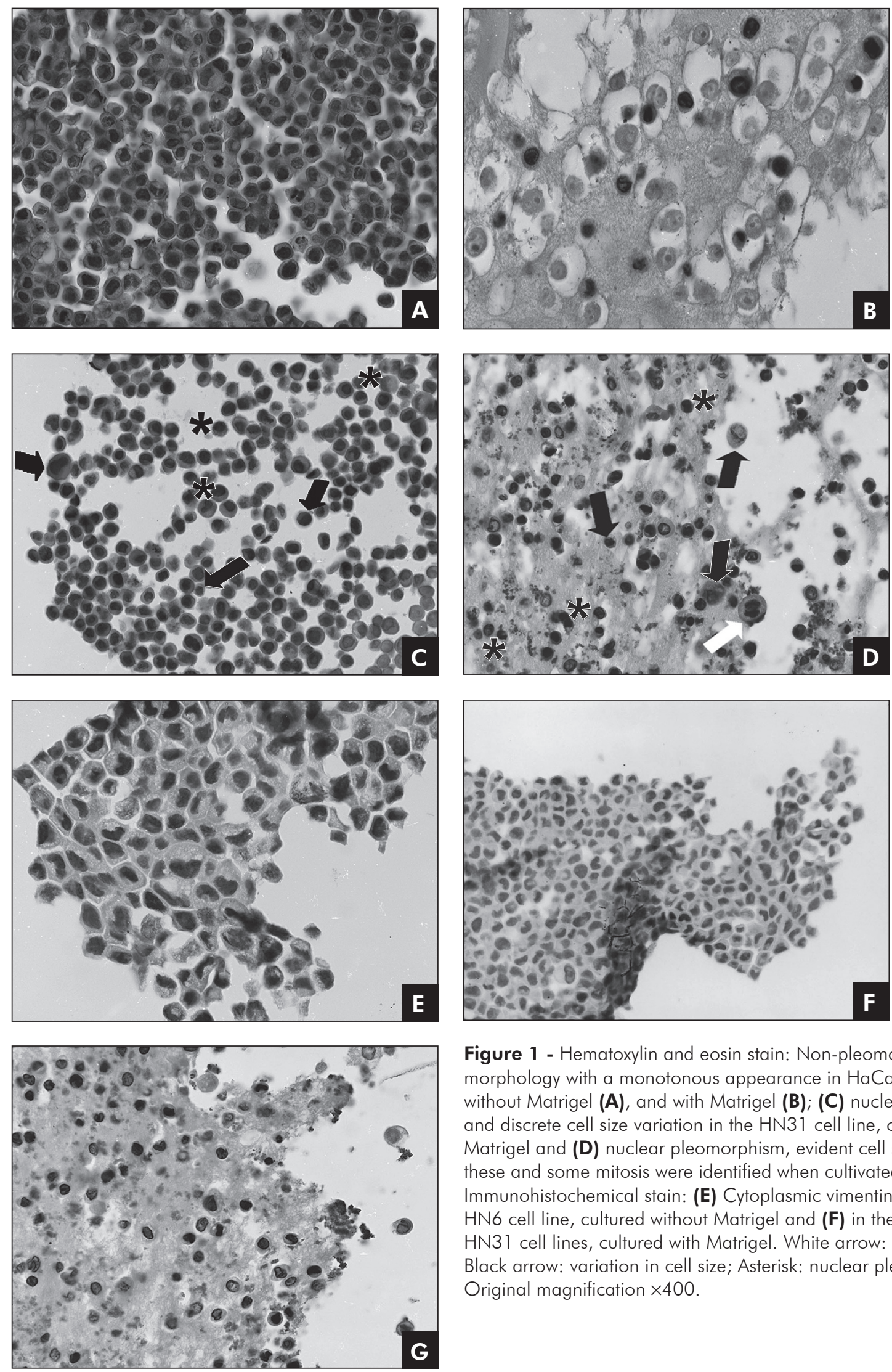

Figure 1 - Hematoxylin and eosin stain: Non-pleomorphic cell morphology with a monotonous appearance in $\mathrm{HaCaT}$ cell line cultured without Matrigel (A), and with Matrigel (B); (C) nuclear pleomorphism and discrete cell size variation in the HN31 cell line, cultured without Matrigel and (D) nuclear pleomorphism, evident cell size variation: these and some mitosis were identified when cultivated with Matrigel. Immunohistochemical stain: (E) Cytoplasmic vimentin expression in the HN6 cell line, cultured without Matrigel and (F) in the HN30 and (G) HN31 cell lines, cultured with Matrigel. White arrow: mitosis figure; Black arrow: variation in cell size; Asterisk: nuclear pleomorphism. Original magnification $\times 400$. 

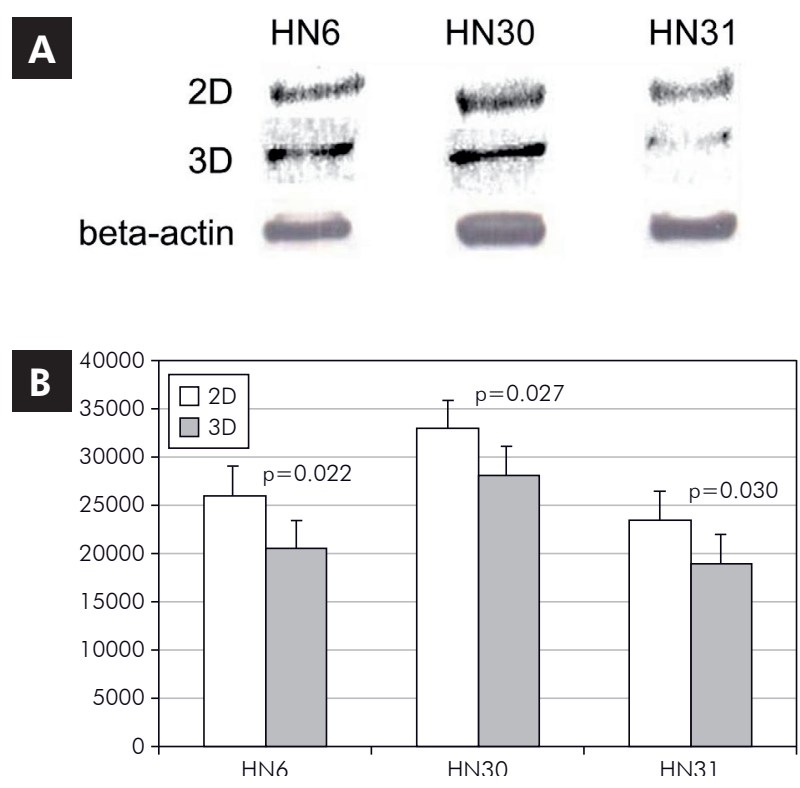

Figure 2 - (A) Bands demonstrated in Western blotting analyses of the HNSCC cell lines, for the antibody investigated (Vimentin). Beta-actin was used to control total volume of each sample. (B) Representative graph of mean values plus standard deviation. Statistically significant differences between each cell line are represented by distinct letters: (a) HN6 - $\mathrm{p}=0.022$; (b) HN30 - $\mathrm{p}=0.027$; (c) HN31 $\mathrm{p}=0.030$.

microenvironments rather than on two-dimensional surfaces. ${ }^{20}$ To mimic the extracellular matrix, a three-dimensional cell culture with Matrigel was used in this study. Matrigel contains components of the human extracellular matrix including laminin, type IV collagen, nidogen, entactin and heparan sulfate. ${ }^{17}$

The present study showed no spindle cells compatible with mesenchymal phenotype in the HNSCC cell lines cultivated with Matrigel. In vivo, this phenotype change is usually described in carcinoma cells at the tumor invasion front, and is associated with more aggressive behavior. ${ }^{8}$ However, characteristics also related to a more aggressive cellular pattern, like evident nuclear pleomorphism, cell size variation and mitosis, were observed in the HNSCC cell lines cultivated with Matrigel. This suggests the ability of this substrate ability to simulate the in vivo microenvironment. ${ }^{21}$

Matrigel probably stimulates growth and retains the cells in an undifferentiated state, which represents an invasive phenotype. ${ }^{22}$ This hypothesis ex- plains the evident vimentin expression in the HN30 and HN31 cell lines after being cultured with Matrigel.

The HN6 cell line has a constitutively activated epidermal growth factor receptor. ${ }^{19}$ Interestingly, among all the studied cell lines cultured without Matrigel, only the HN6 cell line expressed the vimentin protein, suggesting its more aggressive phenotype.

It is known that the vimentin protein contributes to the aggressive pattern of cancer cells because it regulates the interaction between cytoskeletal proteins with cell adhesion molecules. It thereby participates in cell adhesion, migration, invasion and cell signal transduction in tumor cells, tumor-associated endothelial cells and macrophages. Its highly dynamic balances between polymerization and depolymerization, and its complex phosphorylation may serve as the regulation mechanisms for tumor metastasis and cell-cell interactions. Therefore, vimentin is recognized as a hallmark of tumor progression that characterizes highly invasive and metastatic carcinomas. $^{14}$

On the other hand, no immunohistochemical expression and significant decrease in vimentin intracellular levels were noted for the HN6 cell line cultured with Matrigel. Although this vimentin expression could have occurred subsequently, the time spent in the cell culture was probably not enough to promote it for this cell line. This time dependence can be an important factor to be considered when working with three-dimensional culture models. In this study, it was observed that Matrigel degraded after 3 days. Thus, if the cells remain in contact with the substrate for a longer time, the experiment would become 2D (conventional method of cultivation) and important data from the cell-environment interaction could be lost.

Another important finding demonstrated through immunohistochemistry was that, for the cell lines that did express vimentin (HN6 cultured without Matrigel; and HN30 and HN31 cultured with Matrigel), only part of these cells showed vimentin staining. This could be explained by the different degrees of cellular differentiation. In agreement, Gilles et al. ${ }^{13}$ demonstrated that only part 
of the epithelial mammary cells express vimentin, while the most differentiated cells do not. Furthermore, the lack of vimentin expression in the $\mathrm{HaCaT}$ cell line confirms its immortalized but nontumorigenic nature. ${ }^{23}$

\section{Conclusions}

Data from this study indicate differences be-

\section{References}

1. Schaaij-Visser TB, Brakenhoff RH, Leemans CR, Heck AJ, Slijper M. Protein biomarker discovery for head and neck cancer. J Proteomics. 2010 Set 10;73(10):1790-803.

2. Scully C, Bagan J. Recent advances in Oral Oncol. Oral Oncol. 2007 Feb;43(2):107-15.

3. Crowe D, Hacia J, Hsieh C, Sinha U, Rice H. Molecular pathology of head and neck cancer. Histol Histopathol. 2002;17(3):909-14.

4. Hanahan D, Weinberg RA. The hallmarks of cancer. Cell. 2000 Jan 7;100(1):57-70.

5. Ruiz P, Gunthert U. The cellular basis of metastasis. World J Urol. 1996;14(3):141-50.

6. Boyer B, Valles AM, Edme N. Induction and regulation of epithelial-mesenchymal transitions. Biochem Pharmacol. 2000 Oct 15;60(8):1091-99.

7. Hay ED. The mesenchymal cell, its role in the embryo, and the remarkable signaling mechanisms that create it. Dev Dyn. 2005 Jul;233(3):706-20.

8. Birchmeier C, Birchmeier W, Brand-Saberi B. Epithelialmesenchymal transition in cancer progression. Acta Anat. 1996;156(3):217-26.

9. Araujo VC, Pinto Junior DS, Sousa SO, Nunes FD, Araujo NS. Vimentin in oral squamous cell carcinoma. Eur Arch Otorhinolaryngol. 1993;250(2):105-9.

10. Ivaskaa J, Pallarib HM, Nevoa J, Eriksson JE. Novel functions of vimentin in cell adhesion, migration, and signaling. Exp Cell Res. 2007 Jun 10;313(10):2050-62.

11. Mendez MG, Kojima SI, Goldman RD. Vimentin induces changes in cell shape, motility, and adhesion during the epithelial to mesenchymal transition. FASEB J. 2010 Jun;24(6):183851.

12. Taki M, Kamata N, Yokoyama K, Fujimoto R, Tsutsumi S, Nagayama M. Down-regulation of Wnt-4 and up-regulation of Wnt-5a expression by epithelial-mesenchymal transition in human squamous carcinoma cells. Cancer Sci. 2003 Jul;94(7):593-7. tween two and three-dimensional cell culture models. Therefore, the distinct expression of vimentin found for each of the cell lines studied, as well as its strict relation to the extracellular matrix, indicates that the behavior of the HNSCC cell lines can be influenced by both the extracellular microenvironment and the culture conditions.

13. Gilles C, Polette M, Zahm JM, Tournier JM, Volders L, Foidart JM, et al. Vimentin contributes to human mammary epithelial cell migration. J Cell Sci. 1999 Dec;112(Pt 24):461525.

14. Liu LK, Jiang XY, Zhou XX, Wang DM, Song XL, Jiang HB. Upregulation of vimentin and aberrant expression of E-cadherin/beta-catenin complex in oral squamous cell carcinomas: correlation with the clinicopathological features and patient outcome. Mod Pathol. 2010 Feb;23(2):213-24.

15. LeBleu V, MacDonald B, Kalluri R. Structure and Function of Basement Membranes. Exp Biol Med. 2007 Oct;232(9):1121-9

16. Boudreau N, Bissel M. Extracellular matrix signaling: interation of form and function in normal and malignant cells. Curr Opin Cell Biol. 1998 Oct;10(5):640-6.

17. Kleinman HK, McGarvey ML, Hassell JR, Star VL, Cannon $\mathrm{FB}$, Laurie GW, et al. Basement membrane complexes with biological activities. Biochemistry. 1986 Jan 28;25(2):312-8.

18. Schmeichel K, Bissell M. Modeling tissue-specific signaling and organ function in three dimensions. J Cell Sci. 2003 Jun 15;116(Pt 12):2377-88.

19. Cardinali M, Pietraszkiewicz H, Ensley J, Robbins K. Tyrosine phosphorylation as a marker for aberrantly regulated growthpromoting pathways in cell lines derived from head and neck malignancies. Int J Cancer. 1995 Mar 29;61(1):98-103.

20. Griffith L, Swartz M. Capturing complex 3D tissue physiology in vitro. Nat Rev Mol Cell Biol. 2006 Mar;7(3):211-24.

21. Kujan O, Oliver RJ, Khattab A, Roberts SA, Thakker N, Sloan P. Evaluation of a new binary system of grading oral epithelial dysplasia for prediction of malignant transformation. Oral Oncol. 2006 Nov;42(10):987-93.

22. Hughes CS, Postovit LM, Lajoie GA. Matrigel: a complex protein mixture required for optimal growth of cell culture. Proteomics. 2010 May;10(9):1886-90.

23. Ren Q, Kari C, Quadros MR, Burd R, McCue P, Dicker AP, et al. Malignant transformation of immortalized $\mathrm{HaCaT}$ keratinocytes through deregulated nuclear factor kappaB signaling. Cancer Res. 2006 May;66(10):5209-15. 